Energetic Processing of Interstellar Silicate Grains by Cosmic Rays

E. M. Bringa, S. O. Kucheyev, M. J. Loeffler, R. A. Baragiola, A. G. G. M. Tielens, Z. R. Dai, G. Graham, S. Bajt, J. Bradley, C. A. Dukes, T. E. Felter, D. F. Torres, W. van Breugel

March 29, 2007

The Astrophysical Journal 
This document was prepared as an account of work sponsored by an agency of the United States Government. Neither the United States Government nor the University of California nor any of their employees, makes any warranty, express or implied, or assumes any legal liability or responsibility for the accuracy, completeness, or usefulness of any information, apparatus, product, or process disclosed, or represents that its use would not infringe privately owned rights. Reference herein to any specific commercial product, process, or service by trade name, trademark, manufacturer, or otherwise, does not necessarily constitute or imply its endorsement, recommendation, or favoring by the United States Government or the University of California. The views and opinions of authors expressed herein do not necessarily state or reflect those of the United States Government or the University of California, and shall not be used for advertising or product endorsement purposes. 


\section{Energetic processing of interstellar silicate grains by cosmic rays}

E.M. Bringa ${ }^{1}$, S.O. Kucheyev ${ }^{1}$, M. J. Loeffler ${ }^{2}$, R.A. Baragiola ${ }^{2}$, A.G.G.M. Tielens ${ }^{3}$, Z.R. Dai ${ }^{4}$, G. Graham ${ }^{4}$, S. Bajt ${ }^{1}$, J. Bradley ${ }^{4}$, C. A. Dukes ${ }^{2}$, T.E. Felter ${ }^{1}$, D.F. Torres ${ }^{5}$, and W. van Breugel ${ }^{4}$

${ }^{1}$ Lawrence Livermore National Laboratory, Livermore, CA 94550, USA

${ }^{2}$ University of Virginia, Laboratory for Atomic and Surface Physics, Thornton Hall B113, Charlottesville VA 22903 USA

${ }^{3}$ NASA Ames Research Center, MS 245-3, Moffett Field, CA 94035

${ }^{4}$ Institute of Geophysics and Planetary Physics, Lawrence Livermore National Laboratory, Livermore, CA 94550, USA

${ }^{5}$ Institut de Ciencies de l'Espai, Campus UAB, 08193 Barcelona, Spain

\section{Abstract}

While a significant fraction of silicate dust in stellar winds has a crystalline structure, in the interstellar medium nearly all of it is amorphous. One possible explanation for this observation is the amorphization of crystalline silicates by relatively "low" energy, heavy ion cosmic rays. Here we present the results of multiple laboratory experiments showing that single-crystal synthetic forsterite $\left(\mathrm{Mg}_{2} \mathrm{SiO}_{4}\right)$ amorphizes when irradiated by $10 \mathrm{MeV} \mathrm{Xe} \mathrm{X}^{++}$ions at large enough fluences. Using modeling, we extrapolate these results to show that $0.1-5.0 \mathrm{GeV}$ heavy ion cosmic rays can rapidly ( $\sim 70$ Million yrs) amorphize crystalline silicate grains ejected by stars into the interstellar medium. 


\section{Introduction}

Interstellar dust grains, while comprising only $\sim 1 \%$ of the mass in the interstellar medium, are important because they catalyze the formation of many gas phase molecules, in particular $\mathrm{H}_{2}$ (Hollenbach \& Salpeter 1971) which allow the cooling and collapse of molecular clouds and the formation of stars and planets. High-energy radiation and particles from hot stars, supernovae or active black holes can alter the physical properties of dust grains and thereby affect their role in these processes. Understanding the composition, characteristics, origin and evolution of ISM dust is thus a key question in astrophysics (Draine 2003).

Most of the dust is formed by condensation in the atmospheres of old stars, which eject a significant fraction of their material back into the ISM. Silicates, such as those producing terrestrial planets, are injected from oxygen-rich asymptotic giant branch (AGB) stars, red supergiants, and supernovae (Tielens 2005). The fraction of crystalline silicates observed in stellar winds covers a large range (0.05-0.75; Sylvester et al 1999; Molster et al. 1999). The resulting fraction of crystalline silicates injected into the interstellar medium by red giant and supergiant stars is then estimated to be 0.15 (Kemper et al. 2004; Waters 2004). Specifically, the infrared spectra of the red giants and supergiants reveal the spectroscopic signature of the $\mathrm{Mg}$ rich end members of the olivine and pyroxene families, forsterite $\left(\mathrm{Mg}_{2} \mathrm{SiO}_{4}\right)$ and enstatite $\left(\mathrm{MgSiO}_{3}\right)$. In contrast, the interstellar $9.7 \mu \mathrm{m}$ silicate feature reveals no evidence for crystalline silicates and, hence, after entering the ISM, crystalline silicates ejected by stellar sources are rapidly amorphized by some mechanism. The upper limit for the crystalline fraction of silicates in the interstellar medium is $\sim 1 \%$ (Kemper, Vriend \& Tielens 2004; 2005).

Previous laboratory studies have focused on simulating amorphization of complex silicates by low velocity shocks, using irradiation with low energy (keV) ions. Amorphization has been 
shown in several experiments using 4-50 keV ions at fluences of $10^{15}-10^{17}$ ions $/ \mathrm{cm}^{2}$ (Demyk et al 2001, Jaeger et al 2003; Brucato, Strazzulla, Baratta \& Colangeli 2004). However, this processing is inefficient, because in a $200 \mathrm{~km} / \mathrm{s}$ shock $(0.2 \mathrm{keV} / \mathrm{amu})$ the range of the ions is much smaller than the size of most interstellar grains. Ions of much higher energy, which can penetrate even the largest interstellar grains, pervade the ISM in the form of cosmic rays $(>1$ $\mathrm{MeV} /$ nucleon). However, up till now, processing of dust by such swift ions has been dismissed (Day 1977; Brucato, Strazzulla, Baratta \& Colangeli 2004; Watson \& Salpeter 1972) because it is believed that the accumulated energy deposition by CR cannot reach the values needed for amorphization (Jaeger et al 2003; Brucato, Strazzulla, Baratta \& Colangeli 2004; Demyk et al 2004). In this respect, most of the CR flux consists of $\mathrm{H}$ and $\mathrm{He}$ ions, but $\mathrm{MeV} \mathrm{He}$ shows no evidence for amorphization of silicates even for very high ion fluences (Day 1977). However, it is well known that the fluence/energy deposition required for amorphization with keV ions where elastic atomic interactions dominate - cannot simply be extrapolated to cosmic ray energies - where electronic excitations dominate energy deposition (Ziegler 2003). Indeed, while fast light ions such as $\mathrm{H}$ and He do not typically form amorphous tracks in silicates, swift heavy ions (such as Fe) do, a phenomenon that has been studied for decades (Fleischer, Price \& Walker 1975) and applied to dating and advanced material modification. Heavy cosmic rays $(\mathrm{Fe})$ are less abundant in the ISM than light cosmic rays $\left(\mathrm{H}, \mathrm{He}\right.$ ) by a factor of $\sim 10^{-4}$ (Wiebel-Sooth, Biermann \& Meier 1998 and discussion in Sect. 4). However, at $1 \mathrm{GeV}$, an Fe ion deposits $\sim 10^{4}$ times more energy per unit path length in silicates (Ziegler 2003) than do protons, and lattice defect formation for swift heavy ion is due to electronic energy loss. Therefore, the cumulative damage in interstellar dust by swift heavy ions can rival or even well exceed that by light ions. 
Here we demonstrate, using both experiments and modeling, that heavy cosmic ray ions are the dominant processing agents of dust in the diffuse ISM.

This paper is organized as follows. First, in Section 2, we describe the experimental setup, the choice of the material used, and the methods used to analyze and quantify the results, which are described in Section 3. We discuss our findings in Section 4, which begins with a critical analysis of why CR processing of Galactic ISM dust is indeed important (Section 4.1). For this we estimate the ISM CR spectrum using a 'leaky box' model (Section 4.1.1), and, using phenomenological electronic energy deposition models (Section 4.1.2), we demonstrate that our laboratory experiments at $0.08 \mathrm{MeV} /$ nucleon (but with a large electronic energy loss of $\sim 4.5$ $\mathrm{keV} / \mathrm{nm}$ ) can be confidently extrapolated to the relevant (relatively low) CR energies at 1-100 $\mathrm{MeV} /$ nucleon, where amorphization is most efficient. We then end this section with estimates of the interstellar dust grain amorphization timescale (Section 4.1.3) and the expected fraction of interstellar crystalline silicates. In the subsequent section we compare these results with other possible amorphization processes such as ion bombardment by interstellar shocks (Section 4.2), the lifecycle of crystalline silicates (Section 4.3), and the amorphization of dust grains in the Solar system (Section 4.4) and stellar debris disks (Section 4.5). We then conclude (Section 5) that CR processing of ISM dust is indeed important in our own Galaxy, that it may explain the amorphization of crystalline silicates, and that it is likely to be an even more important effect in forming (starburst) galaxies, which have much higher cosmic ray fluxes due to $10-1000$ times larger star formation rates and emerging active black holes (Torres et al. 2004).

\section{Method}

To investigate the effects of cosmic rays on crystalline silicates, we irradiated samples of undoped forsterite $\left(\mathrm{Mg}_{2} \mathrm{SiO}_{4}\right)$ single crystals with triply ionized, $10 \mathrm{MeV}$ xenon ions. After 
irradiation with specific fluences, we analyzed these samples using Rutherford Backscattering/channeling (RBS/C) spectrometry, infrared (IR) reflection spectroscopy, and transmission electron microscopy (TEM) of electron transparent cross sections of the irradiated forsterites. In this section, we briefly discuss the experimental techniques.

\subsection{Ion irradiation and RBS/C spectrometry}

The LLNL 4 MV ion accelerator was used for ion bombardment and for RBS/C analysis in single alignment conditions. Samples of laboratory grown undoped forsterite $\left(\mathrm{Mg}_{2} \mathrm{SiO}_{4}\right)$ single

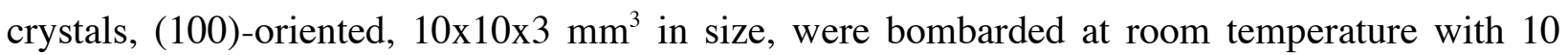
$\mathrm{MeV}{ }^{132} \mathrm{Xe}^{3+}$ ions using a flux of flux of $1-2 \times 10^{10}$ ions $/ \mathrm{cm}^{2} / \mathrm{s}$ to a fluence range of $5 \times 10^{11}-$ $6 \times 10^{13} \mathrm{~cm}^{-2}$. Ion incidence was at an angle of $\sim 7^{\circ}$ relative to the surface normal to minimize channeling. At this energy, $86 \%$ of the total stopping power (energy loss per unit path length) is electronic (4.5 keV/nm, decreasing with penetration depth as the ion slows down [Ziegler 2003]). Furthermore, in this case the xenon ions have a projected range of $\sim 2.4 \mu \mathrm{ms}$ in forsterite (Ziegler, Biersack \& Littmark 1985), and, hence can only alter a near-surface layer with thickness close to the projected ion range.

RBS/C (Chu, Mayer \& Nicolet 1978) is a well-established quantitative technique for the analysis of the elemental composition and crystallinity of the surface and the outer few micrometers of solids. Typically, in $\mathrm{RBS} / \mathrm{C}$, a beam of $0.1-3.0 \mathrm{MeV} \mathrm{He}$ ions is directed at the sample. The flux and energy distribution of the elastically backscattered particles, measured under a given angle, gives information on the structure of the sample. We characterized all our samples after irradiation by $\mathrm{RBS} / \mathrm{C}$ with $2 \mathrm{MeV}^{4} \mathrm{He}^{+}$ions incident along the [100] direction and backscattered at $164^{\circ}$. Damage buildup curves were analyzed with the defect overlap model of cylindrical tracks (Gibbons 1972), where, in the simplest case of so-called zero overlap, the 
relative concentration of defects is $\left[1-\exp \left(-\pi R_{\text {track }}{ }^{2} \mathrm{D}\right]\right.$, where $\mathrm{D}$ is ion fluence, and $R_{\text {track }}$ is the effective radius of an amorphous track that each impinging ion creates along its path in the crystal. Note that the "bulk" samples used here should require roughly the same amorphization fluence as sub- $\mu \mathrm{m}$ grains (Bringa \& Johnson 2004). However, grains smaller than $10 \mathrm{~nm}$ may not only amorphize but be destroyed completely by a single CR impact (Bringa \& Johnson 2004; Berthelot et al 2000; Toulemonde, Assmann, Gruner \& Trautmann 2002).

\subsection{Transmission Electron Microscopy - Sample Preparation and Measurements}

After ion irradiation, 100-nm thick forsterite sections were prepared using a FEI 237 Dualbeam focused ion beam (FIB)/field emission scanning electron microscope (FESEM) and FEI Nova 600 dual beam FIB/FESEM instruments (Graham et al 2004). The extracted cross-sections were attached to the edge of $\mathrm{Cu}$ grids using Pt deposition gas in the FIB. The electron transparent FIB sections were examined using a $300 \mathrm{keV}$ Philips CM300 field emission transmission electron microscope (TEM) equipped with an Oxford Instruments solid-state energy dispersive X-ray (EDX) spectrometer, EmiSPEC spectral processing software and Digital Micrograph (v. 3.3.1) imaging software. Bright-field and dark-field imaging, electron diffraction and EDX spectroscopy were used to investigate compositions and mineralogy of the extracted sections. All images were recorded using a slow-scan charge-coupled-device (CCD) camera (2048 x 2048 pixels).

\subsection{Infrared spectroscopy}

Laboratory infrared spectroscopy cannot only examine the structure of a material but, more importantly, allows for comparison with astronomical observations. While the infrared spectra of 
crystalline materials are typically dominated by a few well-defined narrow bands, amorphous materials are characterized by broad and structure-less bands (Dorschner 1999; Jaeger et al 1998). In the present study, we examined the ion-irradiated samples using IR reflection spectroscopy. The infrared measurements were made using an attenuated total infrared reflectance (ATR) in a Thermo-Nicolet 670 Nexus Spectrometer operating at $4 \mathrm{~cm}^{-1}$ resolution. The beam path and sample compartment were purged continuously with dry air. The ATR used a $\mathrm{ZnSe}$ substrate and an angle of incidence of $45^{\circ}$. The reflectance was normalized to the total reflectance of the $\mathrm{ZnSe}$ prism without a sample. The sampling depth was less than $\sim 2 \mu \mathrm{m}$. Note that, since the index of refraction of crystalline forsterite in the $10-12 \mu \mathrm{m}$ region is even higher than that of $\mathrm{ZnSe}$, the spectra appear different from what would be expected in a transmission experiment. ATR measurements of the samples taken 1 month after irradiation and repeated $\sim 5-6$ months after irradiation produced the same ATR spectra within errors.

\section{Results}

Astronomical IR data demonstrate unambiguously that crystalline silicates are the-magnesiumrich end-members olivine (forsterite, $\mathrm{Mg}_{2} \mathrm{SiO}_{4}$ ) and pyroxene (enstatite, $\mathrm{MgSiO}_{3}$ ) (Sylvester et al. 1999; Molster et al. 2002a,b,c, Waters 2004). This study is focused on the irradiation effects on forsterite; we expect that enstatite with a similar composition will behave similarly. The RBS/C spectra of irradiated forsterite samples show a progressive increase in the RBS/C yield with increasing fluence [Fig. 1(a)], which is due to amorphization, revealed by TEM and infrared absorption spectroscopy data discussed below. If we assume that each ion forms a cylindrical track of amorphous material along its path, full amorphization of the near-surface layer is reached as the fluence increases and the tracks overlap. Using the model described above (Gibbons 1972), we derive an effective track radius of (2.8 \pm 0.6$) \mathrm{nm}$ [Fig. 1(b)]. 
TEM studies revealed that the sample was amorphized up to a depth nearly half the projected ion range (2400 nm, Ziegler 2003) at a fluence of $3 \times 10^{13} /$ ions $/ \mathrm{cm}^{2}$, as shown in Fig. 2(a)-(b). TEM results are similar to previous reports for ion-induced amorphization of $\mathrm{SiO}_{2}$ in the electronic collision regime (Meftah et al. 1994), and of enstatite at the border of electronic and elastic collision regimes (Jaeger et al 2003). Chemical analysis of the amorphous region by energy dispersive X-ray spectroscopy and X-ray photoelectron spectroscopy did not indicate any change in composition.

Attenuated total infrared reflectance (ATR) spectra for different ion fluences are shown in Fig. 3(a). The transition from crystalline forsterite - characterized by narrow spectral features - to an amorphous silicate structure - evidenced by a broad structureless absorption band (Jaeger et al 2003; Brucato, Strazzulla, Baratta \& Colangeli 2004; Demyk et al 2004) - occurs at the same fluences as indicated by the RBS/C analysis in Fig. 1.

\section{Discussion}

\subsection{Amorphization by cosmic rays in the interstellar medium}

The rate at which crystalline silicates are amorphized in the interstellar medium can be determined by convolving the energy dependent flux of cosmic rays with the "damage" level produced by ions of a given energy. We discuss this in more detail below.

\subsubsection{The interstellar cosmic ray spectrum}

To estimate the CR flux experienced by interstellar grains, we note that it is not sufficient to consider the high energy CR flux ( $>1 \mathrm{GeV} /$ nucleon) measured on Earth, since the lower energy CRs - that should dominate amorphization in the ISM - are inhibited from entering the Solar system by the magnetic field in the solar wind. The cosmic ray spectrum can be described using 
a leaky box model for the propagation and escape of galactic CRs (Ip \& Axford 1985). The flux, $\Phi(\mathrm{E})$, as a function of total energy per particle, $\mathrm{E}$, is then given by:

$$
\left.\Phi=\mathrm{C} \mathrm{E}^{0.3} /\left(\mathrm{E}+\mathrm{E}_{\mathrm{o}}\right)^{3} \quad\left(\mathrm{~cm}^{2} \mathrm{~s} \mathrm{sr} \mathrm{GeV}\right)^{-1} \quad \text { (Eq. } 1\right)
$$

where $\mathrm{C}$ is a constant that, as we show below, can be determined by a fit to the high energy $\mathrm{CR}$ flux measured on Earth, and the scaling factor $\mathrm{E}_{\mathrm{o}}$ sets the level of low energy cosmic rays (Webber \& Yushak 1983).

Because molecular abundances in interstellar clouds depend ultimately on the cosmic ray ionization rate, molecular observations provide a direct handle on the low energy cosmic ray flux (Guellin et al. 1977; Wootten et al. 1979; McCall et al. 2003). While earlier studies report cosmic ray ionization rates of $2 \times 10^{-17} / \mathrm{s}$ for molecular clouds, recent observations of $\mathrm{H}_{3}{ }^{+}$and other species have converged on a cosmic ray ionization rate of $2 \times 10^{-16} / \mathrm{s}$ in the diffuse interstellar medium (Tielens 2005).

Using equation 1, the primary cosmic ray ionization rate can be calculated using the differential ionization cross-section (Opal, Beaty \& Peterson 1972) fit to the experimental data on $\mathrm{H}_{2}$ (Rudd, Kim, Madison \& Gallagher 1985). The primary CR ionization rate as a function of the scaling factor $E_{o}$ describing the low energy cosmic ray ionization flux is then:

$$
\zeta_{p}=5.85 \times 10^{-16}\left(\mathrm{E}_{\mathrm{o}} / 100 \mathrm{MeV}\right)^{-2.56} \mathrm{~s}^{-1}(\mathrm{H}-\text { nuclei })^{-1} \text {. }
$$

Hence, the molecular observations imply $\mathrm{E}_{\mathrm{o}} \sim 120 \mathrm{MeV}$ for $\mathrm{H}$.

The value $\mathrm{C}=1.45\left(\mathrm{~cm}^{2} \mathrm{~s} \mathrm{sr} \mathrm{GeV}-1.7\right)^{-1}$, was obtained by matching Eq. 1 to experimental data at high energies (e.g. E=1 TeV/particle; Wiebel-Sooth, Biermann \& Meier 1998) where modulation by the Solar wind has no influence. The high energy data for protons was taken as $\Phi\left(\mathrm{cm}^{2} \mathrm{~s} \mathrm{sr}\right.$ $\mathrm{GeV})^{-1}=\Phi_{0}[\mathrm{E}(\mathrm{GeV}) /(1000 \mathrm{GeV})]^{-\gamma}$, with $\Phi_{0}=11.5110^{-9}\left(\mathrm{~cm}^{2} \mathrm{~s} \mathrm{sr} \mathrm{GeV}\right)^{-1}$ and $\gamma=2.77$ (WiebelSooth, Biermann \& Meier 1998). 
The flux of Fe CR responsible for amorphization of interstellar silicates is then provided by Eq. 1, with $\mathrm{E}_{\mathrm{o}}$ equal to $56 \times 0.12 \mathrm{GeV} /$ particle $=6.72 \mathrm{GeV} /$ particle and $\mathrm{C}=8.1610^{-4}\left(\mathrm{~cm}^{2} \mathrm{~s} \mathrm{sr}\right.$ $\left.\mathrm{GeV}^{-1.7}\right)^{-1}$. C was obtained as for the $\mathrm{H}$ flux, by matching Eq. 1 to experimental data at $\mathrm{E}=1 \mathrm{TeV}$, with $\Phi_{0}=1.7810^{-9}\left(\mathrm{~cm}^{2} \mathrm{~s} \mathrm{sr} \mathrm{GeV}\right)^{-1}$ and $\gamma=2.6$ (Wiebel-Sooth, Biermann \& Meier 1998). This gives a Fe flux that is $\sim 5.610^{-4}$ smaller than the $\mathrm{H}$ flux. Using earlier experimental data, Leger, Jura \& Omont (1985) assumed a factor of $\sim 1.610^{-4}$ between the $\mathrm{H}$ and Fe fluxes, which would give a slightly lower total Fe flux. Fig. 4 shows the resulting $\mathrm{H}$ and $\mathrm{Fe}$ flux.

\subsubsection{Amorphization by ions at interstellar cosmic ray energies}

While the ion energies in our experiments $(0.08 \mathrm{MeV} /$ nucleon) are in the electronic energy regime, they are lower than relevant cosmic ray energies (1-100 MeV/nucleon). However, based on models for ion damage in solids, validated by experiments on high-energy irradiation of quartz, we can extrapolate our results to the ISM. We have fit the experimental results for several ions bombarding quartz (Meftah et al 1999) to two analytical phenomenological models (Szenes 1997; Tombrello 1994) for track size versus stopping power.

At $\mathrm{keV}$ energies, the energy deposition is dominated by elastic atom-atom collisions. At $\mathrm{MeV}$ energies and above, corresponding to CR's, energy deposition is mostly due to electronic excitations. Specifically, heavy cosmic rays create nm-sized tracks of dense electronic excitations that can be (partly) converted into atomic motion (Bringa \& Johnson 2004; Berthelot et al 2000; Toulemonde, Assmann, Gruner \& Trautmann 2002; Szenes 1997; Tombrello 1994; Meftah et al 1994) and can lead to amorphization in a cylinder of the material forming the track. For ion energies typical for CR's, the cross section to create electronic excitations increases with increasing ion mass (Ziegler 2003). 
To extrapolate the track size measured for $10 \mathrm{MeV}$ Xe-atoms to astrophysically relevant ions and energies, we have used both the "thermal spike" model proposed by Szenes (1997) and the bond-breaking model by Tombrello (1994). We calculated $d E / d x$ vs. $E$ for both Xe and Fe using the SRIM2003 code (Ziegler 2003) for a forsterite density of $3.2 \mathrm{~g} / \mathrm{cm}^{3}$. Therefore, $(d E / d x$ ) and $R_{\text {track }}[(d E / d x)]$ are both known as functions of projectile energy. As shown in figure 5, these models, although formulated differently, predict roughly the same size for the amorphous track. The semi-analytic model by Toulemonde et al (2002) also gives similar results. All these models are expected to give poor results at low $d E / d x$, close to the threshold for track formation, but still agree within a factor of 2 with the experimental data in this $d E / d x$ range. Given that the track size in pure $\mathrm{SiO}_{2}$ scales fairly well with $(d E / d x)_{\text {elec }}$ alone, as shown in Fig. 5, we expect roughly the same amorphous track radius for the same $d E / d x$ for any ion, including Fe ions. We note that the data in Fig. 5 is for a variety of ions in the range $\sim 0.01-3.6 \mathrm{GeV}$ and that its dispersion might be partly due to the "velocity effect" [Meftah et al, 1994], i.e. two different ions with the same $d E / d x$ (but different velocity) produce somewhat different track sizes. Even taking into account this dispersion, one can notice the reasonably good agreement between the different models and experiments, including our results on forsterite, despite the much higher chcemical complexity compared to $\mathrm{SiO}_{2}$. This agreement supports our extrapolation of the track radius to the higher $\mathrm{CR}$ energies in the ISM.

\subsubsection{The interstellar amorphization timescale}

For a given ion energy $E$, there is a flux $4 \pi \Phi(E)$ on each grain. Each ion creates an amorphous

track of area $\pi R_{\text {track }}{ }^{2}$, which depends on the incident energy through $d E / d x(E)$. The amorphization time, $\Delta t_{\text {amorph }}$, of a grain can be estimated as: 


$$
\Delta t_{\text {amorph }}=\left(\int_{E_{\min }}^{E_{\max }} 4 \pi^{2} R_{\text {track }}^{2} \Phi(E) d E\right)^{-1}
$$

It is possible to integrate over multiple $\mathrm{CR}$ species, besides $\mathrm{Fe}$, with $d E / d x$ high enough to produce amorphous tracks, but the flux of these other species is negligible and would not significantly change the amorphization time calculated for Fe alone. For sub- $\mu \mathrm{m}$ grains, even the less energetic cosmic ray $\left(\right.$ at $E_{\text {min }}$ ) will produce an amorphous track across the whole grain. In this limit, $\Delta t_{\text {amorph }}$ is independent of the grain area. Eq. 2 neglects any track overlap, but including it based on a model [Gibbons 1972, Figure 1(b)] further increases the amorphization time by up to a factor of $\sim 4$. This is the number we report below.

Note that the minimum kinetic energy for a CR escaping from a supernova remnant shock, $E_{m i n}$, can be fixed by requiring that it be at least larger than $2 m v_{\text {shock }}^{2}$, where $m$ is the mass of the particle and $v_{\text {shock }}$ the shock velocity (Bell 1978). For shock velocities of the order of $10^{3-4} \mathrm{~km} / \mathrm{s}$, $E_{\min }$ is in the range of $5.2 \mathrm{keV}-0.52 \mathrm{MeV}$ for a proton, and correspondingly larger for heavier ions. For Fe CR, $E_{\min } \sim 0.29-29 \mathrm{MeV}$. Since no tracks form in thin layers for $E>5 \mathrm{GeV}[(d E / d x)<$ $1.5 \mathrm{keV} / \mathrm{nm}]$, we use $E_{\max }=5 \mathrm{GeV}$. Using the flux described above for $E_{\min }=0.03,0.1$ and $1 \mathrm{GeV}$ gives $\Delta t_{\text {amorph }} \sim 65,68.5$ and 180 million yrs. respectively. These times correspond to $\sim 233,230$ and $180 \mathrm{Fe} C \mathrm{CR}$ impacts every million years on a grain of $0.1 \mu \mathrm{m}$ radius.

\subsubsection{The fraction of interstellar crystalline silicates}

Based upon our experiments, we have calculated an exposure time of nearly 70 million yr to completely amorphize crystalline silicates in the ISM. To evaluate quantitatively the crystalline silicate fraction in the ISM, we develop a simple model following McKee (1989) and Kemper, Vriend \& Tielens (2004). Amorphous and crystalline silicate grains are injected into the ISM by stars in the later stages of their evolution and destroyed by sputtering in strong shock waves 
driven by supernova explosions. In addition, dust is lost when new stars are formed. The last process to consider is the amorphization of crystalline grains by cosmic rays. In steady state, the crystalline-to-amorphous mass fraction, $\delta$, is then given by:

$$
\delta=\left(\mathrm{k}_{\mathrm{SF}}+\mathrm{k}_{\mathrm{d}}\right)\left(\mathrm{k}_{\mathrm{CR}}\left(1+\delta_{\mathrm{o}}\right)+\mathrm{k}_{\mathrm{SF}}+\mathrm{k}_{\mathrm{d}}\right)^{-1} \delta_{\mathrm{o}}
$$

under the assumption that sputtering destroys crystalline and amorphous silicates at the same rate. Here, $\mathrm{k}_{\mathrm{SF}}$ is the star formation rate $\left(2 \times 10^{-10} \mathrm{yr}^{-1}\right.$, corresponding to $1 \mathrm{M}_{\text {sun }} / \mathrm{yr}$; McKee 1989), $\mathrm{k}_{\mathrm{d}}$ is the dust destruction rate $\left(2.5 \times 10^{-9} \mathrm{yr}^{-1}\right.$, corresponding to silicate lifetime of $4 \times 10^{8} \mathrm{yr}$; Jones, Tielens \& Hollenbach 1996), $\mathrm{k}_{\mathrm{CR}}$ is the amorphization rate $\left(1.4 \times 10^{-8} \mathrm{yr}^{-1}\right.$, corresponding to $7 \times 10^{7}$ $\mathrm{yr}$-this work) and $\delta_{\mathrm{o}}$ is the fraction of silicates injected into the ISM in crystalline form. With these values, this equation simplifies to:

$$
\delta \sim \mathrm{k}_{\mathrm{d}} / \mathrm{k}_{\mathrm{CR}} \delta_{\mathrm{o}}
$$

Estimates for $\delta_{0}$ vary from $\sim 0.15$ - if AGB stars dominate the dust budget - to $\sim 0.05$ if supernovae are important sources of amorphous silicates (Kemper, Vriend \& Tielens 2004). The estimated fraction of crystalline silicates ranges then from 0.008 to 0.027 , which should be compared to the observed fraction of less than $\sim 0.01$ (Kemper, Vriend \& Tielens 2005a, b).

\subsection{Amorphization by ion bombardment in interstellar shocks}

As mentioned in the introduction several studies have shown silicate amorphization by $\mathrm{H}$ and $\mathrm{He}$ ions at $4-50 \mathrm{keV}$ at fluences representative of fast shocks in the diffuse ISM. However, the volume of a grain affected by $\mathrm{keV}$ ion irradiation is to first order set by the range of the ion. To fully amorphize a grain of $0.1 \mu \mathrm{m}$ radius, $\mathrm{H}(\mathrm{He})$ ions require an energy of 10 (15) keV (Ziegler 2003), corresponding to a $\sim 1,400(850) \mathrm{km} / \mathrm{s}$ shock. In a two-phase ISM, a single supernova remnant irradiates dust in the equivalent of $\sim 60 M_{S U N}$ of interstellar gas with shocks of velocities exceeding $1000 \mathrm{~km} / \mathrm{s}$ (McKee 1989). With a total ISM gas mass of $4.5 \times 10^{9} M_{S U N}$ and an effective 
supernova rate of $8 \times 10^{-3} / \mathrm{yr}$, the timescale to amorphize is $10^{10} \mathrm{yr}$ (McKee 1989). Therefore, high velocity shocks would not amorphize dust grains efficiently.

\subsection{The lifecycle of crystalline silicates}

The lifecycle of interstellar dust starts with the nucleation and growth of high temperature condensates such as silicates at high densities and temperatures in the ejecta from stars, such as Asymptotic Giant Branch stars and supergiants. This ejecta is rapidly mixed with other gas and dust in the ISM. A dust grain cycles many times between the intercloud and cloud phases until it is either destroyed by fast supernova shocks or incorporated into newly formed stars or planetary systems. Observations with the Infrared Space Observatory have revealed that crystalline silicates are abundant in the initial stages of this life cycle - the ejecta from stars - as well as in last stages - circumstellar disks around Herbig AeBe stars and T-Tauri stars, but are absent in the intermediate stages (Sylvester et al. 1999; Kemper et al. 2004; 2005; Malfait et al. 1999; Waters and Waelkens 1998). As our experimental and modeling results demonstrate, crystalline silicates injected into the ISM can be rapidly amorphized by galactic cosmic rays on a timescale that is short compared to other relevant timescales (section 4.1.3). The high abundance of crystalline silicates in circumstellar disks surrounding young stars (Waters and Waelkens 1998) must then reflect subsequent processing of grains in these environments. Recent mid-infrared spectroscopic interferometry on AU-scale-sizes has revealed the presence of a strong gradient in the crystallinity of silicates in the circumstellar disks of the three objects investigated (van Boekel et al. 2004). Likely this reflects the rapid annealing in the hot inner regions of these disks coupled with turbulent diffusion outwards of the crystalline grains.

Parenthetically, we mention here that presolar SiC grains recovered from meteorites at the parts per million level are generally crystalline. These grains are formed in the ejecta of AGB 
stars and other stellar dust sources and after a long and harsh sojourn in the interstellar medium became part of the solar nebula and eventually the parent body of meteorites. While our experiments do not directly pertain to $\mathrm{SiC}$, this material should also amorphize by high-energy ion bombardment. These grains are larger than $10 \mu \mathrm{m}$, and therefore larger than typical interstellar grains and it is unknown whether they were incorporated into meteorites as individual $\mathrm{SiC}$ grains or as components of even larger multi-mineral grains. The extent to which they have been modified after accretion into the meteorite parent body is also unknown but recrystallization by thermal annealing in the solar nebula is possible.

\subsection{Amorphization of Grains in the Solar system}

Crystalline silicates are an abundant component of dust from comets. Likewise, Interplanetary Dust Particles (IDPs) collected in the stratosphere contain silicates (Bradley, Humecki \& Germani 1992; Bradley 2004). It is thus of interest to consider the amorphization of crystalline silicates in the Solar system. IDPs are part of the zodiacal dust, a tenuous disk of small (1-200 $\mu \mathrm{m})$ dust particles orbiting within $5 \mathrm{AU}$ of the Sun. The lifetime of these particles due to the Poynting-Robertson drag has been calculated to be $\sim 10^{5}$ years (Fixsen \& Dwek 2002). Direct estimates of IDP residence times in the solar system have been obtained from observations of implanted solar flare tracks densities and measurements of galactic and solar cosmic-raygenerated radionuclides (Bradley et al. 1984; Nishiizumi et al., 1991). These lifetimes are shorter than the timescale against complete amorphization of $\mu \mathrm{m}$-sized silicates by galactic cosmic rays derived in section 4.1.3, even more so if we consider that low energy cosmic rays are excluded from the solar system by the Solar wind. 


\subsection{Amorphization of Grains in Debris Disks}

More than $15 \%$ of all main sequence stars have a debris (or dusty) disk (Habing et al 1999; Lagrange, Backman \& Artymowicz 2000). A-type stars do not have stellar winds and, hence, the interstellar CR induced crystallization lifetime calculated in section 4.1 .3 can be compared to the dust lifetime in these systems. We estimate the lifetime against the Poynting-Robertson drag as $\sim 10^{5} \mathrm{yr}$ for an A star with $\mathrm{M}_{*}=3 \mathrm{M}_{\mathrm{o}}$. These grains will thus not be amorphized by galactic cosmic rays over their lifetime. Grains dynamically trapped in a resonance with an orbiting planet (Kalas et al. 2005) can be much older and amorphized on a timescale of $\sim 70$ million years by galactic cosmic rays, which could be revealed by infrared spectroscopy of this dust. However, if the lifetime of the system is limited, e.g. $\beta$-Pictoris which is estimated to have an age of only 12 million years, amorphization will not have proceeded very far yet even for dynamically trapped grains.

\section{Conclusion}

Our experimental irradiation studies show that crystalline silicates are readily amorphized by swift heavy ions, in agreement with previous studies on $\mathrm{SiO}_{2}$. Using theoretical models we extrapolated these experimental results to astrophysically relevant energies to show that cosmic rays with energies between 0.1 and $5 \mathrm{GeV}$ will amorphize crystalline silicate grains in the ISM on a timescale of 70 million years. This timescale is much shorter than that between the injection of silicate materials into the ISM by old stars and their incorporation into new stars and planetary systems ( 2500 million yr; Savage \& Sembach 1996), and the destruction of silicates in the ISM (400 million yr; Jones, Tielens \& Hollenbach 1996). We estimate that, in steady state, less than $0.3 \%$ of the crystalline grains injected into the ISM survive amorphization. This is qualitatively consistent with the observed interstellar abundance of crystalline silicates (Kemper 
et al., 2005) as well as the abundance of (isotopically anomalous) presolar crystalline silicates in meteorites and IDPs (Messenger et al., 2003; Floss et al., 2005).

The IR spectra of irradiated silicates lose the narrow features characteristic of crystalline materials and develop a broad featureless band in the 10-12 $\mu \mathrm{m}$ region. This spectral change due to amorphization parallels the emission spectral differences between silicates ejected by old stars and silicate grains observed in the ISM. Thus, cosmic ray irradiation of interstellar silicates affects the opacity of the ISM.

In addition, we have examined the irradiation of silicates in the solar system and in debris disks. For the Solar system, cosmic ray irradiation of zodiacal dust will have little effect since the Poynting-Robertson drag severely limits the grains lifetime in the inner Solar system. Amorphization of debris disks around A-type stars by cosmic rays is also similarly limited by the Poynting-Robertson drag unless the dust is trapped in long-lived resonances with planetary companions. Infrared spectroscopic studies of such systems may provide information on the lifetime of these grains.

We note that other consequences of CR irradiation are the destruction of small grains (Bringa \& Johnson 2004) and the synthesis and ejection of complex molecules formed on the grain surface. CRs will also restructure the grain surfaces, which may affect the formation efficiency of $\mathrm{H}_{2}$ as suggested by Monte Carlo simulations revealing that the temperature range for high efficiency $\mathrm{H}_{2}$ formation is larger for heterogeneous than for crystalline surfaces (Cuppen \& Herbst 2005). Since we find that the amorphization time scale is so short, nearly all dust grains in the ISM will have been processed and the $\mathrm{H}_{2}$ and subsequent star formation will be maximized (positive feedback). 
Energetic ion impacts may have been particularly important in the early universe during the formation and co-evolution of galaxies and their central, galactic black holes (Di Matteo, Springel \& Hernquist 2005; Silk \& Rees 1998). Those environments are characterized by both rapid injection of freshly synthesized (crystalline) silicates by short-lived massive stars as well as high supernova rates (e.g., high cosmic ray fluxes) and jets of energetic particles from the central black hole. The crystalline-to-amorphous silicate fraction in these environments is then a balance between the injection and the amorphization process and, based upon our experimental and theoretical studies, observations could then be used to deduce the flux of energetic particles in these environments, which is hard to determine otherwise.

Recently, the spectroscopic signature of crystalline silicate was recognized in the spectra of a sample of deeply embedded Ultra Luminous InfraRed Galaxies (ULIRGs; Spoon et al. 2005). This class of ULIRGs likely reflects a brief, early phase in the evolution of ULIRGs in which star formation and AGN activity has not yet had time to process and disperse the nuclear gas and dust. The presence of copious amounts of crystalline silicates in these starburst environments suggests that the dust has been injected very recently ( $<70$ Million yrs), presumably by massive stars formed in the star burst, and therefore has not yet been amorphized by CR ions. This is consistent with ULIRG starburst age estimates of 10 - 100 Million years (Genzel et al. 1998).

Acknowledgments The authors would like to thank G. Strazzulla, M. Toulemonde, C. McKee, and R.E. Johnson for useful discussions. N. Teslich (LLNL) and M. Bernas (FEI Company) are thanked for performing the FIB work. This work was performed under the auspices of the U.S. Department of Energy (DOE) by the University of California, Lawrence Livermore National 
Laboratory (LLNL) under Contract No. W-7405-Eng-48. The project 04-ERD-059 was funded by the Laboratory Directed Research and Development Program at LLNL.

The studies at the University of Virginia and LLNL were supported in part by grants (to RAB and JPB) from NASA's Cosmochemistry and Origins of the Solar System programs.

Author Information: Correspondence and request for materials should be addressed to E.M.B (ebringa@1lnl.gov) or W.v.B. (wil@igpp.llnl.gov). 


\section{References}

Bell, A. R. 1978, MNRAS, 182, 443

Berthelot, A. et al. 2000, Phil. Mag. A 80, 2257

Black, J. H. \& Dalgarno, A. 1973, ApJ, 184, L101

Bradley, J. P., 2004, In Treatise on Geochemistry, 1, 689-711, Eds A. Davis, H. D. Holland and K. K.Turekian.

Bradley, J. P.; Brownlee, D. E.; Fraundorf, P., 1984, Science, 226, 1432

Bradley, J. P., Humecki, H. J. \& Germani, M. S. 1992, ApJ, 394, 643

Bringa, E. M. \& Johnson, R. E. A. 2004, ApJ, 603, 159

Brucato, J. R., Strazzulla, G., Baratta, G., \& Colangeli, L. A. 2004, A\&A, 413, 395

Cuppen, H. M. \& Herbst, E. 2005, MNRAS, 361, 565

Chiar, J. \& Tielens, A. G. G. M. 2005, ApJ, submitted

Chu, W. K., Mayer, J. W. \& Nicolet, M. A., 1978, Backscattering Spectroscopy (New York, Academic)

Day, K. L. 1977, MNRAS, 178, 49

Demyk, K. et al. 2001, A\&A, 368, L38

Demyk, K., d'Hendecourt, L., Leroux, H., Jones, A. P. \& Borg, J. 2004, A\&A, 420, 233

Di Matteo, T., Springel, V. \& Hernquist, L, 2005, Nature 433, 604

Dorschner, J, 1999, in Formation and Evolution of Solids in Space, Edited by J. Mayo Greenberg and Aigen Li, (Kluwer Academic Publishers), p.229

Draine, B. T. 2003, ARA\&A 41, 241

Fixsen, D. J. \& Dwek, E. 2002, ApJ, 578, 1009 
Fleischer, R. L., Price, P. B. \& Walker, R. M. 1975, Nuclear Tracks in Solids (Univ. California Press, Berkeley, 1975)

Floss, C., Stadermann, F. J., Bradley, J. P., Dai, Z. R., Bajt, S., Lea, A. S., GCA, 70, 2371.

Genzel, R. et al. 1998, ApJ, 498, 579

Gibbons, J. F. 1972, Proc. IEEE, 60, 1062

Graham, G. et al. 2004, Lunar Planet. Sci. 35, Abstr. \#2044

Guelin, M., Langer, W.D., Snell, \& R.L.Wootten, H.A., 1977, ApJ, 217, L165

Habing, H. J.; Dominik, C.; Jourdain de Muizon, M.; Kessler, M. F.;

Laureijs, R. J.; Leech, K.; Metcalfe, L.; Salama, A.; Siebenmorgen, R.; Trams, N. 1999, Nature, 401,456

Hollenbach, D. \& Salpeter, E. E. 1971, ApJ 163, 155

Ip, W.-H. \& Axford, W.I. 1985, A\&A, 149, 7

Jaeger, C, Fabian, D., Schrempel, Dorschner, J., Henning, Th. \&. Wesch, W. 2003, A\&A, 401, 57

Jaeger, C., Molster, F. J., Dorschner, J., Henning, Th., Mutschke, H. \&

Waters, L. B. F. M. 1998, A\&A, 339, 904

Jones, A.P., Tielens, A.G.G.M. \& Hollenbach, D., 1996, ApJ, 469, 740

Kalas, Paul; Graham, James R.; Clampin, Mark , 2005, Nature, 435, 1067

Kemper, F., Vriend, W. J. \& Tielens, A. G. G. M. 2004, ApJ, 609, 826 (2005, erratum ApJ, 633, 534)

Koike, C.; Chihara, H.; Tsuchiyama, A.; Suto, H.; Sogawa, H.; Okuda, H. 2003, A\&A, 399 (http://www.kyoto-phu.ac.jp/labo/butsuri/data/Koike03AA/Fo100.csv)

Lagrange, A.-M.; Backman, D. E.; Artymowicz, P., 2000, Protostars and 
Planets IV, eds. Mannings, V., Boss, A.P., Russell, S. S, (Tucson:

University of Arizona Press), p. 639

Leger, A., Jura, M. \& Omont, A. 1985, A\&A, 144, 147

Le Petit, F., Roueff, E. \& Herbst, E. 2004, A\&A, 417, 993

Malfait, K., Waelkens, C., Waters, L. B. F. M., Vandenbussche, B.,

Huygen, E. \& de Graauw, M. S. , 1998, A\&A, 332, L25

McCall, B. et al. 2003, Nature, 422, 500

McKee, C.F. 1989, In Interstellar dust (eds. Allamandola, L.J. \& Tielens, A.G.G.M. Proc. IAU Symposium 135, 431 (Kluwer, Dordrecht)

Messenger, S., Keller, L. P., Stadermann, F. J., Walker, R. M., Zinner, E., 2003, Science, 300, 105.

Meftah A. et al. 1994, Phys. Rev. B, 49, 12457

Molster, F.J. et al. 1999, Nature, 401, 563

Molster, F. J., Waters, L. B. F. M., Tielens, A.G.G.M. \& Barlow, M. J. 2002a, A\&A, 382, 184

Molster, F. J., Waters, L. B. F. M., \& Tielens, A. G. G. M., 2002b, A\&A, 382,222

Molster, F. J., Waters, L. B. F. M., Tielens, A. G. G. M., Koike, C. \&

Chihara, H., 2002c, A\&A, 382, 241

Nishiizumi, K., Arnold, J. R., Fink, D., Klein, J., \& Middleton, R. 1991,

E\&PSL, 104, 315

Opal, C. B., Beaty, E. C. \& Peterson, W. K. 1972, Atomic Data 4, 209

Rudd, M. F., Kim, Y. K., Madison, D. H. \& Gallagher, J. W. 1985, Rev. Mod. Phys. 57, 965

Savage, B.D. \& Sembach, K.R. 1996, ARA\&A, 34, 279 
Silk J. \& Rees, M. 1998, A\&A 331, L1

Spoon, H. W. W. et al. 2006, ApJ, 638, 759

Sylvester R. J. et al. 1999, A\&A 352, 587

Szenes, G. 1997, Nucl. Instr. Meth. Phys. Res. B 122, 530

Tielens, A.G.G.M. 1998, ApJ, 499, 267

Tielens, A.G.G.M., 2005, Physics and Chemistry of the Interstellar Medium, (Cambridge; Cambridge University Press)

Tombrello, T. A. 1994, Nucl. Instr. Meth. Phys. Res. B 94, 424

Torres, D.F., Reimer, O., Domingo-Santamaria, E., Digel, S.W. 2004, ApJ Letters, 607, 99

Toulemonde, M., Assmann, W., Gruner, F., Trautmann, C. 2002, Phys. Rev. Lett. 88, 057602

van Boekel, R.; Min, M.; Leinert, Ch. , et al., 2004, Nature, 432, 479

Waters, L. B. F. M. \& Waelkens, C. 1998, ARA\&A, 36, 233

Waters, L. B. F. M.. 2004, Astrophysics of Dust, ASP Conference Series, Vol. 309, Edited by A. N. Witt, G. C. Clayton and B. T. Draine., p.229

Watson, W. D. \& Salpeter, E. E. 1972, ApJ, 174, 321

Webber, W. R. \& Yushak, S. M. 1983, ApJ, 275, 391

Wiebel-Sooth, B., Biermann, P.L. \& Meyer, H. 1998, A\&A, 330, 389

Wootten, A., Snell, R., Glassgold, A.E., 1979, ApJ 234, 876

Ziegler, J. F., SRIM 2003.26 (www.srim.org).

Ziegler, J. F., Biersack, J. P. \& Littmark, U. 1985, The Stopping and Range of Ions in Solids (New York, Pergamon) 


\section{Figure Captions}

Fig. 1: (a) Selected RBS/C spectra illustrating the accumulation of lattice disorder in forsterite single crystals irradiated with $10 \mathrm{MeV} \mathrm{Xe}$ ions at room temperature at different fluences. The spectrum for a fluence of $3 \times 10^{13} / \mathrm{cm}^{2}$ coincides with the random spectrum.

Normalized RBS/C yields fit to a track-overlap amorphization model (Gibbons 1972) giving an effective ion track radius of $2.8 \mathrm{~nm}$.

Fig. 2: Cross-sectional bright-field (a) and dark-field (b) TEM images of single crystal forsterite irradiated at room temperature with $10 \mathrm{MeV}$ Xe ions to a dose of $3 \times 10^{13} / \mathrm{cm}^{2}$. Shown in (c) and (d) are selected-area electron diffraction patterns taken from the left and right parts of the specimen, respectively, confirming the ion-induced structural modification from the initial single crystal to a completely amorphous material.

Fig. 3: (a) ATR infrared spectra of forsterite samples irradiated at different fluences. Astronomical emission spectra showing a broad amorphous band in the ISM (Chiar \& Tielens 2005) and crystalline peaks for dust in the ejecta of the red giant, AC Her (Molster, Waters, Tielens \& Barlow 2002) A laboratory spectrum of polycrystalline forsterite (Koike et al 2003) is also shown for comparison.

Fig. 4: Cosmic ray flux of $\mathrm{H}$ and $\mathrm{Fe}$ versus total energy (energy/particle), for both the unmodulated [Wiebel-Sooth et al. 1998] and modulated [Webber\&Yushak 1983] case.

Fig. 5: Amorphous track radius vs. electronic stopping power $d E / d x$. Experimental data points are for forsterite (this work) and quartz (Meftah A. et al. 1994). The lines are results of the thermal spike (Szenes 1997) and bond-breaking (Tombrello 1994) model predictions. The $d E / d x$ range for $0.1-10 \mathrm{GeV} F e$ cosmic rays in forsterite is also shown. 


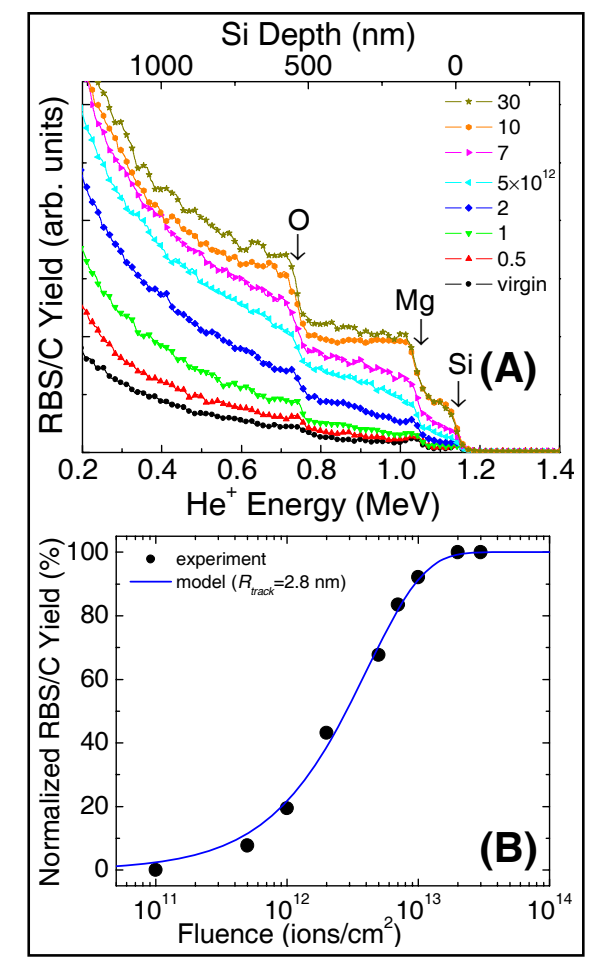

Fig. 1 


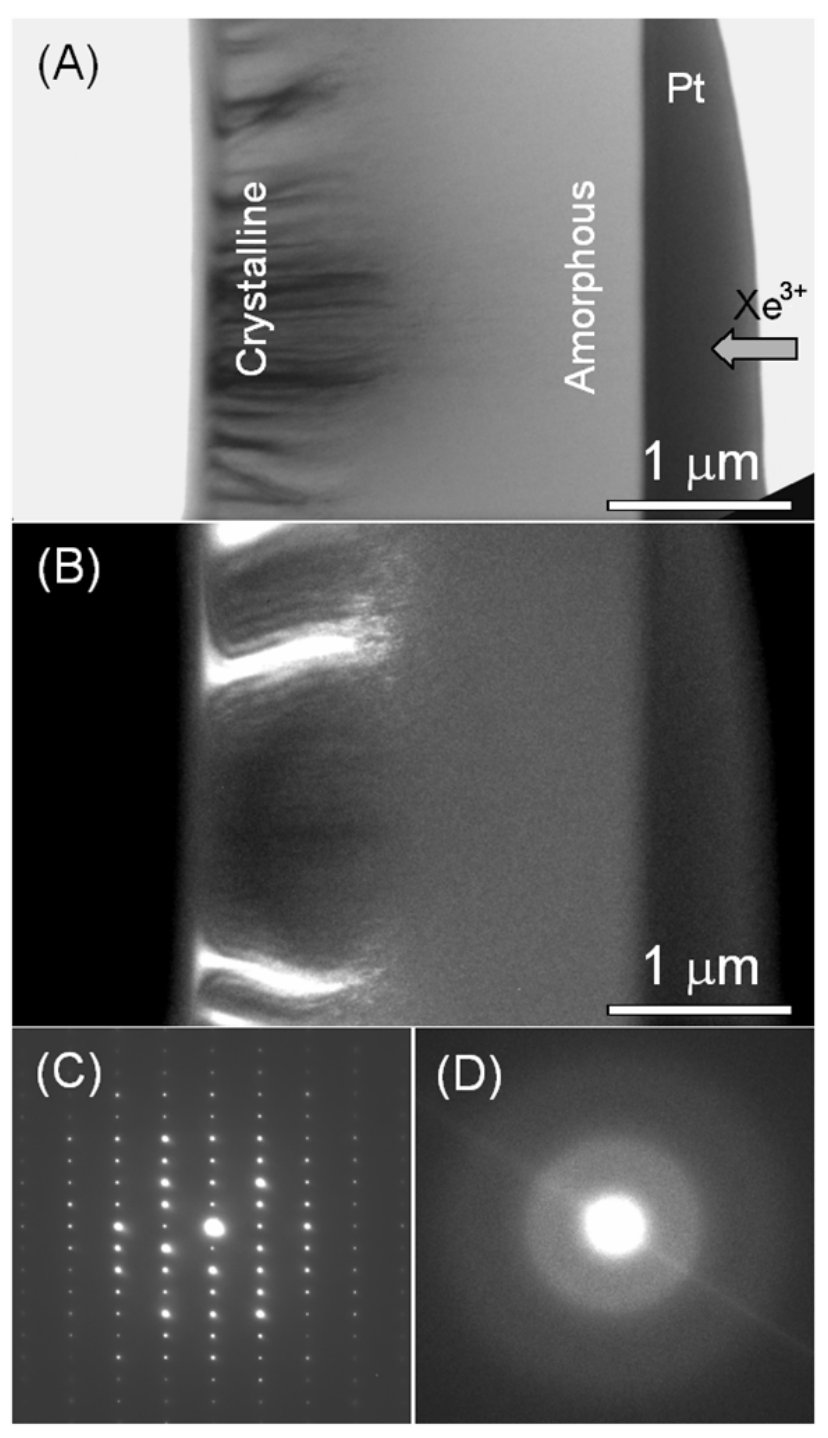

Fig. 2 


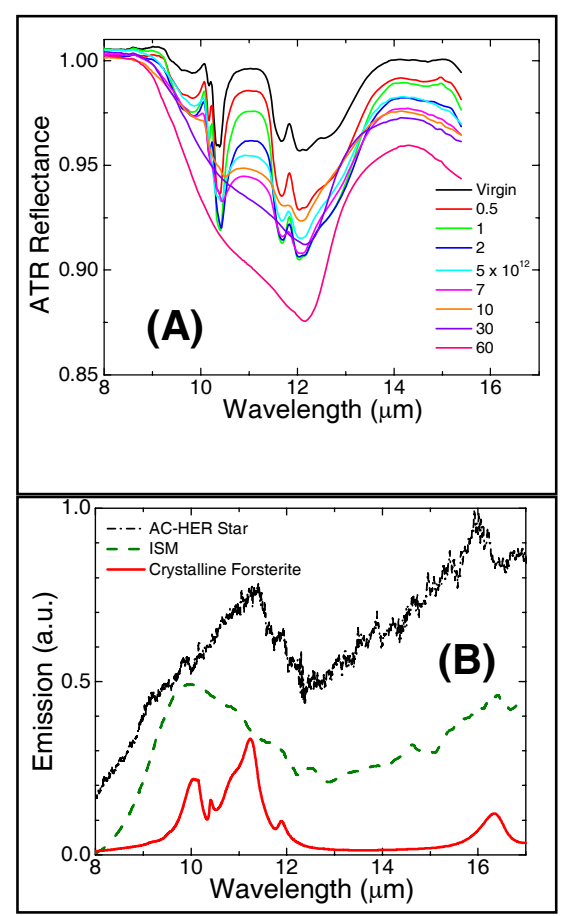

Fig. 3 


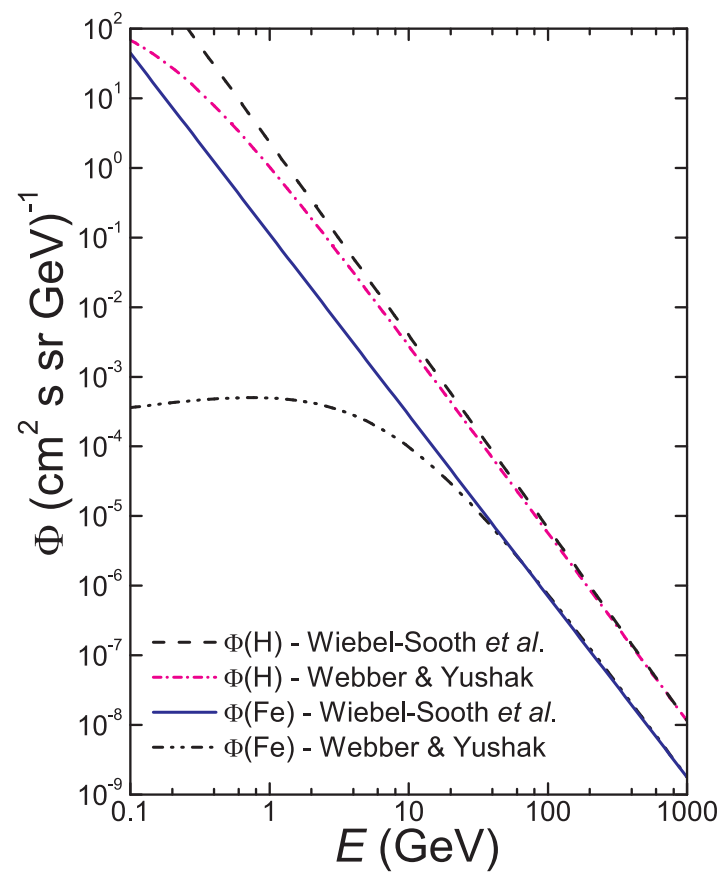




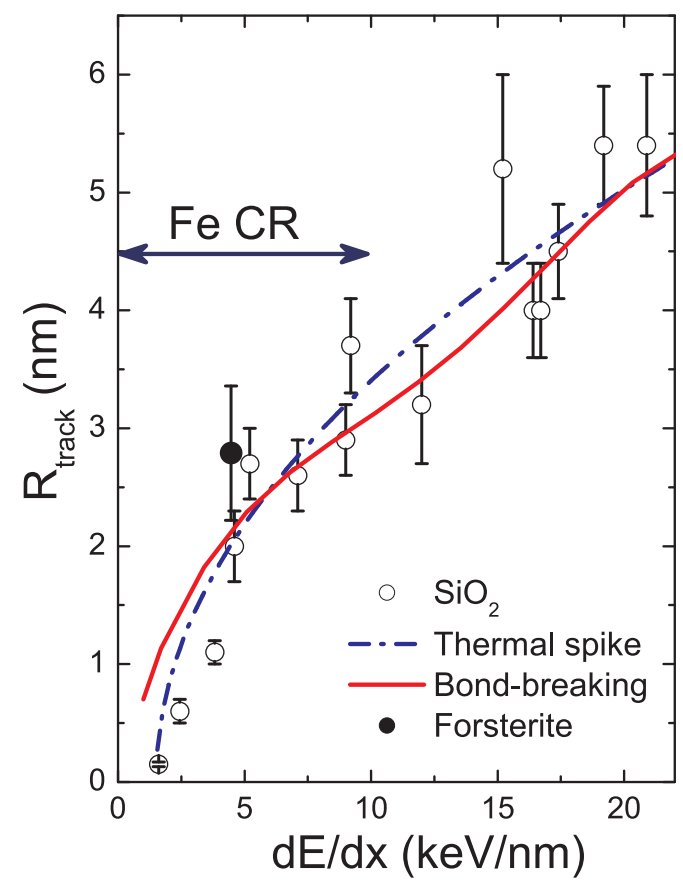

